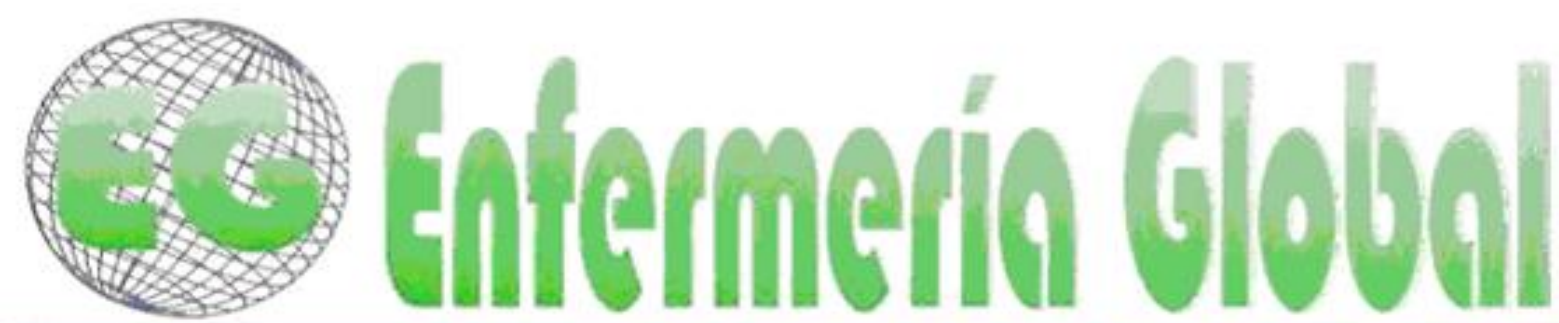

ISSN 1695-6141 Revista electrónica trimestral de Enfermería

$N^{\circ} 40$

www.um.es/eglobal/

Octubre 2015

DOCENCIA - INVESTIGACIÓN

\title{
"Ahí me aguanté mi dolor". Contexto sociocultural y económico de mujeres con síndrome con coronario residentes en Cali y otros municipios del Valle del Cauca
}

"There, I endured my pain". Sociocultural and economic context of women with coronary syndrome in Cali and other municipalities in the Valle del Cauca

\section{*Valencia Micolta, Susana Genith *Rodríguez Torres, Estela}

*Enfermera.Mg SP. E-mail: svalenci09@gmail.com **Universidad Santiago de Cali. Colombia.

\begin{abstract}
Palabras clave: salud de la mujer; enfermedad coronaria; factores socioeconómicos: determinantes sociales de la salud; factores culturales (fuente: DeCS. BIREME)
\end{abstract}

Keywords: women's health, coronary disease: socioeconomic factors; social determinants of health; cultural factors (source: MeSH, NLM)

\section{RESUMEN}

La investigación forma parte del "Estudio Multicéntrico, Comparación de la caracterización de los síntomas de angina en la mujer en ocho ciudades de Colombia". Aborda las condiciones de desigualdad en empleo, educación, género y etnia, situaciones de violencia y desplazamiento forzoso, factores fisiológicos que desencadenan una serie de síntomas y aparición del síndrome coronario, angina e infarto agudo de miocardio.

Objetivo: Determinar aspectos socioculturales y económicos que influyen en la aparición de síntomas de angina en mujeres de Cali y municipios del Valle del Cauca

Material y método. Estudio descriptivo con abordaje cualitativo, en 23 mujeres. Con criterios de haber experimentado molestia, dolor o sensación de disconfort torácico, hemodinámicamente estables, arteriografía coronaria con lesión de vasos mayor de 70\%, y postsíndrome coronario agudo. Las entrevistas son analizadas con técnica de análisis de contenido de Klipendorf y Teoría de Síntomas Desagradables de Lenz y colaboradores

Resultados. Los síntomas desagradables, tienen relación con antecedentes fisiológicos y factores influyentes del ambiente físico, familiar y social. Emergen categorías acerca de responsabilidades laborales y domésticas, asumir roles, apoyo a la familia y conviviendo con sus dificultades, viviendo con incertidumbre e inseguridad, relaciones con el entorno, cumpliendo con el tratamiento.

Conclusión. El modo de vida en las mujeres que padecen de síndrome coronario agudo es un factor influyente en el agravamiento de los síntomas. Las mujeres están expuestas a experiencias y entornos desventajosos en condiciones de vulnerabilidad e inequidad. 


\section{ABSTRACT}

The research is part of the Multicenter Research "Comparative characterization of the symptoms of angina in women in eight cities of Colombia."

It addresses the conditions of inequality in employment, education, gender and ethnicity, violence and forced displacement, physiological factors that trigger a variety of symptoms and the appearance of coronary syndrome, angina and acute myocardial infarction.

Objective: To determine socio-cultural and economic factors that affect the onset of symptoms of angina in women from Cali and Valle del Cauca municipalities.

Material and methods: Descriptive qualitative study in 23 women with background of having experienced discomfort, chest pain or discomfort, hemodynamically stable, coronary arteriography vessel injury increased $70 \%$, and post-acute coronary syndrome. The interviews are analyzed with the content analysis technique of Klipendorf and the Theory of Unpleasant Symptoms by Lenz and colleagues.

Results: Unpleasant symptoms are related to physiological background and factors of physical, family and social environment. Findings related to work and domestic responsibilities, assuming roles, family support and coping with their difficulties, living with uncertainty and insecurity, relationships with the environment, complying with treatment.

Conclusion: The way of life in women with acute coronary syndrome, is an influential factor in the worsening of symptoms. Women are exposed to disadvantageous experiences and environments in conditions of vulnerability and inequality.

\section{INTRODUCCIÓN}

En el mundo se estima en 17 millones de personas las que han muerto por enfermedades cardiovasculares (ECV), y son la principal causa de defunción en la región de las Américas ${ }^{(1,2) ;}$ para el año 2007 fueron responsables casi 1,6 millones de muertes $^{(3)}$ y fueron dominadas por las enfermedades isquémicas del corazón en el $43 \%$ de las muertes por ECV, afectando a ambos sexos ${ }^{(4)}$ y más del $80 \%$ se producen en países de ingresos bajos y medios, entre los cuales se encuentra Colombia.

Según el Departamento Nacional de Estadística (DANE) el grupo de las enfermedades cardio-vasculares (CCV), ocupa la primera causa de mortalidad en ambos sexos, la enfermedad isquémica del corazón ocupa en el sexo femenino la primera causa de mortalidad en todas las edades ${ }^{(5)}$. En el año 2011 se registró la más alta proporción de fallecimientos por ECV correspondiente al $25.4 \%$, el riesgo de morir aumenta con la edad, presentándose unas tasas de mortalidad superiores a la tasa nacional en uno de los departamentos como es el Valle del Cauca ${ }^{(6)}$; el cual cuenta con 4.532.378 habitantes, en el 2005 se reportó que la mortalidad por ECV como el infarto agudo de miocardio (IAM)tienen una tasa de 29, enfermedad cardiaca hipertensiva 21, otras enfermedades cerebro vasculares 22, hipertensión esencial primaria 11, y enfermedad isquémica crónica del corazón de 11 por cada 100.000 habitantes $^{(7)}$. Para el año 2006 la enfermedad crónica isquémica del corazón presentó 406 casos, siendo la décima causa de mortalidad. Las muertes relacionadas con las principales enfermedades cardiovasculares, han permanecido estables, en el 2007 hubo un incremento en tasa relativa del 18\% comparado con el año 1998 de la cardiopatía isquémica $^{(8)}$.

En el proceso de transición demográfica, el envejecimiento de la población va en aumento, y los cambios en el estilo de vida están repercutiendo directamente en el perfil epidemiológico de la población; tal es el reflejo en el aumento de las Enfermedades No Transmisibles Crónicas (ECNT), este grupo se incluye la 
Enfermedad Cardiaca y los Síndromes Coronarios Agudos (SCA), cabe recordar que quien padece este tipo de enfermedades ha estado expuesto a experiencias y entornos desventajosos a través del tiempo se acumula a lo largo de toda la vida aumentando el riesgo de enfermedad y muerte prematura ${ }^{(9)}$. De allí que existen inequidades y desigualdades sociales y de salud que determinan el riesgo y vulnerabilidad de padecer una enfermedad. Entendiéndose la vulnerabilidad como un proceso dinámico establecido por la interacción de los elementos que la componen, tales como edad, raza, etnia, pobreza, escolaridad, soporte social y presencia de agravantes a la salud. Se admite que cada persona posee un umbral de vulnerabilidad que cuando se sobrepasa aparece la enfermedad ${ }^{(7)}$. Por esta razón es necesario identificar oportunamente los riesgos y la vulnerabilidad, para brindar cuidado permanente, prevenir mayores complicaciones y secuelas graves que afecten el bienestar individual, familiar e inclusive la muerte.

En el estudio se analizan situaciones relacionadas con los factores influyentes, fue necesario articular los factores de riesgo, teniendo en cuenta aquellos que hacen más vulnerable a la mujer. Teniendo en cuenta aquellos determinantes, que según la comisión de "Determinantes Sociales de la Salud (CDSS) son clasificados como estructurales, que a su vez configuran los determinantes intermediarios" ${ }^{(10)}$. El nivel educativo de la mujer influye en el posicionamiento en la escala laboral y el ingreso; también aquí se ubican la etnia y las relaciones de género, desde esta perspectiva, las condiciones de desigualdad la ha llevado a adoptar hábitos y estilos de vida que sumado a factores biológicos y psicosociales tienen efectos perjudiciales en su bienestar afectando la calidad de vida, en la cual aparecen y ocurren eventos como el síndrome coronario, la angina y el infarto agudo de miocardio. Dichos estudios generan evidencias que orientan a repensar en estrategias y acciones para cambiar este perfil de morbi-mortalidad mediante la aplicación del conocimiento sobre los factores de riesgo modificables y la resiliencia ${ }^{(11)}$; los resultados permiten aportar estrategias para el cuidado de la mujer desde un modelo de bienestar en promoción de la salud y prevención del riesgo de manera oportuna y generar propuestas que se articulen a diseños y gestión de políticas públicas que determinen para amplios sectores de la población, la diferencia entre sobrevivir o morir, entre disfrutar la experiencia de la vida y no someterse a condiciones penosas de existencia ${ }^{(12)}$, en el trasegar de las vida cuando la mujer adulta asume y afronta responsabilidades con una alta carga emocional.

El síndrome coronario representa en la población femenina factores influyentes que están presentes en la aparición de los síntomas, los cuales la mujer una vez que los padece debe afrontarlos. Es necesario reconocer las características del contexto donde se desenvuelven, que incluyen diferentes aspectos: entre estos está el panorama en materia de empleo y posicionamiento de las mujeres en un mercado laboral que económicamente permitan por lo menos satisfacer las necesidades básicas y la relación con la accesibilidad a servicios de salud. La Organización Panamericana de la Salud ${ }^{(13)}$ enfatiza que se debe incorporar la visión de género en las políticas y estrategias de salud para dar cuenta cabal de la realidad, considerando que la equidad en salud se asocia coherentemente con principios de justicia social y de derechos humanos.

Desde esta perspectiva para la atención de salud la equidad de género implica que los recursos y los servicios se asignen respectivamente de acuerdo con las necesidades específicas de hombres y mujeres según las particularidades de dichos grupos. En general se puede decir que la mujer vallecaucana representa etnias afro, 
indígena y mestiza producto de asentamientos desde la época de la colonia, especialmente cuando los negros son traídos al territorio para el trabajo en la agricultura y para desempeñarse en el servicio doméstico, "fenómeno que hace parte del epicentrismo dominante que históricamente ha ejercido Cali, municipio con mayoría de gente negra al igual que la mayor parte de los municipios del Valle geográfico del rio Cauca, por lo menos hasta 1920"(14).

También existe otro aspecto que ha estado presente desde principios del siglo XX hasta la actualidad, como es el caso de la violencia en determinadas regiones producto del conflicto armado en el que las mujeres han tenido que migrar forzosamente con sus hijos, convirtiéndose en mujeres cabeza de hogar, cuyo desempeño en la sociedad lo muestra el estudio de Guevara $^{(15)}$, en el cual da cuenta de las características de los tres grupos étnicos que predominan en la región como son: las mujeres indígenas, que al llegar a su nuevo habitat crean y generan mecanismos de resistencia y de inclusión en condición desplazadas, pero siempre luchando, tratando de conservar su identidad familiar, regional y de género, pues el conflicto armado también atraviesa la vida individual familiar y colectiva que dejan marca en las personas, fragmentando y polarizando los referentes identitarios. En el caso de las afrodescendientes cuyas familias nuclear y extensa son numerosas, establecen redes sociales como medio de solidaridad para rodearse en caso de necesidades especialmente para la crianza de los hijos, y se desenvuelven en actividades idénticas a las de las personas ya establecidas en el territorio anteriormente, y por último el grupo de las mestizas, siguen manteniendo comunicación con sus parientes fuera de la ciudad, pero igual desempeñan funciones del servicio doméstico y trabajos de economía informal; cabe resaltar que cuando se revisan otras investigaciones la situación es igual en otros municipios ${ }^{(15)}$.

Sumado a la situación mencionada anteriormente, otro elemento indispensable y que tiene que ver con el bienestar es el empleo, cuya remuneración es necesaria para satisfacer las necesidades básicas y la relación directa que guarda con la afiliación a un régimen de seguridad social de salud. En el Valle del Cauca el $60 \%$ de los trabajadores sufren de problemas relacionados con baja calidad de empleo por la insatisfacción del ingreso y de la jornada laboral, en los últimos años la proporción de hombres ocupados en actividades informales ha disminuido de forma continua, mientras que la tasa de informalidad femenina se ha mantenido alta y estable.

También es claro que usualmente las mujeres sufren un mayor nivel de subempleo ${ }^{(16)}$, pudiendo acceder a servicios de salud por medio del sistema integrado de servicios y beneficios (SISBEN), implicando una serie de procesos administrativos para lograr el servicio en períodos de tiempo prolongados; situación que lleva de manera alterna a hacer uso de prácticas populares de salud, como aquellos saberes adquiridas de generación en generación a través de las denominadas medicinas caseras y lo que ofrecen las redes de curanderos(as) y boticarios, como parte de la identidad colectiva propia de los sectores populares generando en las personas grandes beneficios en sus vidas cotidianas y universos simbólicos ${ }^{(17)}$ pues existen diversas situaciones que llevan a que la mujer minimice el problema cuando parece que tienen que ver con las obligaciones y responsabilidades como mujer cabeza de hogar, madre, abuela, suegra, consejera, afrontar situaciones de violencia e inseguridad, además de carencias económicas; en el desempeño de estos roles deja de lado su autocuidado ampliando una fuerte conexión con lo espiritual, prácticas de carácter religioso acudiendo a cultos para pedir por su salud, negociar por medio de promesas y el uso 
de medicina popular, solamente cuando la situación se torna grave y crónica acuden a las instituciones.

La Teoría de los Síntomas Desagradables incluye tres componentes ${ }^{(18)}$ que se deben indagar: los síntomas que la persona experimenta, los factores influyentes que afectan la naturaleza de la experiencia del síntoma, y las consecuencias. Se afirma que los factores psicológicos, fisiológicos y situacionales pueden interactuar unos con otros y sus relaciones con los síntomas, existe relación entre los factores influyentes que consideran variables fisiológicas, psicológicas y las situacionales que se han estudiado. Se considera que cuando hay apoyo social los factores situacionales son un buen amortiguador del estrés cuando se experimenta el síntoma. Cuando las interacciones sociales están influenciadas negativamente alteran el estado fisiológico, experimentando ansiedad, y depresión con modificación de las actividades cotidianas. La aplicación de la teoría permite interpretar y comprender las dimensiones del síntoma como experiencia negativa, cuáles son los factores influyentes y las consecuencias, para orientar las intervenciones del cuidado de enfermería.

\section{MATERIAL Y MÉTODO}

La investigación se realizó en el período de 2010-2011. Es un estudio descriptivo con abordaje cualitativo, aplicando la técnica análisis de contenido ${ }^{(19,20)}$. A partir de las unidades de registro y unidades de contexto permiten identificar las categorías, según los tres niveles de la semántica: sintáctico que se refiere al análisis de la superficie del texto, el semántico se explora el significado de palabras y frases situación; y el pragmático que enfoca el lenguaje como comportamiento de un sujeto en su contexto, reflejando los significados de la vivencia de una situación y que explican los comportamientos asociados ${ }^{(20)}$, desde una perspectiva emic. Para determinar el rigor metodológico del estudio se tuvo en cuenta los criterios de credibilidad, auditabilidad y transferibilidad ${ }^{(21)}$.

El estudio contó con la aprobación de los comités de Ética de las Instituciones comprometidas con el desarrollo de la investigación, teniendo en cuenta los principios éticos $^{(22)}$ de confidencialidad y anonimato, participación voluntaria y reserva de los participantes, quienes ingresaron al estudio una vez diligenciaron el consentimiento informado. Para la muestra se aplicó el criterio de saturación de datos ${ }^{(23)}$, participaron 25 mujeres que consultaron en dos instituciones de la ciudad de Cali, que brindan atención de alta complejidad, cumpliendo con los siguientes criterios de inclusión: que hayan experimentado molestia, dolor o sensación de disconfort en el tórax; que al momento de las entrevista estén hemodinámicamente estables, con arteriografía coronaria con lesión mayor de $70 \%$ de uno o varios vasos o que se encuentre post síndrome coronario agudo, y que además conserven procedencia geográfica de la cuidad en la que se encuentran hospitalizadas.

Se realizaron entrevistas en profundidad, a partir de una guía semiestructurada, se transcribe y simultáneamente se analizan los datos con la técnica de análisis de contenido $^{(19)}$, partiendo de las expresiones a través del lenguaje verbal y corporal que permitió identificarlas e interpretan los datos desde la conformación de unidades de muestreo ${ }^{(24)}$, que incluyen el relato general de las experiencias vividas y sentidas por las participantes en relación con las dimensiones de los síntomas desagradables, los factores que lo determinan y las consecuencias de dicha experiencia. Una vez codificada la información se constituyeron las unidades de registro y de contexto, identificando y analizando los datos que permiten comprender los significados del 
comportamiento ${ }^{(25)}$, la semántica y la sintáctica expresada en los códigos sustantivos, e interpretada desde la perspectiva de la emic; considerando la particularidad del género en nuestro caso particular: las mujeres de la región del Valle del Cauca. Finalmente descritas en categorías, subcategorías y taxonomías sintetizando, contextualizando y recontextualizando ${ }^{(19)}$ el fenómeno desde la teoría de Lenz y col. como fundamento teórico del cuidado. Sin embargo, vale la pena resaltar las reflexiones que necesariamente deben hacerse al momento del análisis, y al hablar del componente de factores influyente, las investigadoras interpretan desde el contexto de la Salud Pública lo correspondiente a aquellos factores determinantes con el propósito de repensar en las acciones desde la promoción de la salud y la prevención de la enfermedad. Además al tratarse de la mujer implica abordar desde la conceptualización de Género y las reflexiones epistémicas desde un marco interpretativo e histórico social especialmente cuando de nuestro contexto colombiano se trata.

\section{RESULTADOS}

Dentro de los factores influyentes se encuentran los determinantes situaciones que incluyen: la influencia del ambiente familiar, social, laboral, condiciones de vidaacceso a servicios, y el ambiente físico (calor, humedad, luz, calidad de aire). Se enuncian las categorías correspondientes, según la teoría de Lenz, y en cada una de ellas, las subcategorías desde el enfoque pragmático expresado en el lenguaje como comportamiento de los sujetos en su contexto. Las 25 mujeres participantes del estudio están en edad entre 45 y 75 años; pertenecen a la etnia mestiza y afrodescendiente. En cuanto a su estado civil: el $39.1 \%$ son viudas, $16.2 \%$ viven en unión libre, $14 \%$ separadas, $19.5 \%$ solteras y $11.2 \%$ son casadas. El $91.3 \%$ tienen algún grado de escolaridad, siendo primaria completa el mayor nivel logrado, y $8.7 \%$ analfabetas iletradas. Respecto a su ocupación $17.4 \%$ pensionadas, $13 \%$ trabajadoras y el $8.7 \%$ son desempleadas y en todas las estudiadas el $52.2 \%$ son amas de casa, esta última característica coincide con los hallazgos en grupo de mujeres que participaron en el estudio en el municipio de Girardot ${ }^{(26)}$, encontrando algunas características de modos de vida similares en zonas geográficas de las riberas colombianas, descritas a continuación:

\section{Categoría 1. Cumpliendo con las responsabilidades laborales y domésticas}

Las participantes se desenvuelven en contextos de trabajo formal e informal de manera independiente siendo la mayoría en oficios domésticos, en el lugar del trabajo presentan signos y síntomas de disconfort, pero siguen cumpliendo con sus labores domésticas debido a que de su remuneración depende el sostenimiento del hogar:

"Sí, sí, cuando estaba trabajando pues me tocaba: barrer, trapear, lavar baños, sacudir, lavar paredes (Se queda pensativa y respira profundo, afirma con la cabeza). Entonces, yo me fui a trabajar y todo el día lo pasé con el dolorcito, el dolorcito (se toca repetidamente el pecho con la mano empuñada)".

"El primero que me dio, en la empresa, que ahí fue donde me di cuenta. Yo luchaba... con ese malestar y eso fue aquí en urgencias porque yo me vine... entonces yo le decía a la enfermera jefe, que eran las dos tostadas que se me habían quedado atrancadas y yo trabajando y yo estaba morada y yo no me había dado cuenta..." 
"Y que tiene que terminar el almuerzo. Porque mire que usted tiene que..., porque yo trabajo con lencería, que tengo que entregar un mantel..., que tengo reunión del colegio de la niña...que la plata para pagar los servicios y todo eso..."

Ante la presencia del evento la mujer puede decirse a sí misma que no vale la pena preocuparse, pero cuando el fenómeno se repite se va agotando la capacidad de resistir y aguantar, y en situación de extrema gravedad sí acuden en busca de ayuda.

“... No eso es, a veces que ya me conocen, y dicen ve... llamemos a doña xxxx. A hacer aseo, pues si a veces, pongamos últimamente yo me he negado, por esa vaina del dolor".

"Trabajé en un colegio. A mí me tocaba todo, a mi me tocaba ir al banco, me tocaba de todo, papelería todo eso me tocaba. Hasta que me pensioné y fuera de eso trabajé dos años más después de pensionada. Yo trabajé toda mi vida allá... no trabajé en otra parte sino ahí".

Cabe destacar que algunas mujeres identifican su rol de "amas de casa", actividad que ha sido un oficio de tiempo completo sin ningún reconocimiento en el hogar y como trabajo se mantiene disponible como mano de obra barata. Como lo expresan:

"Vea que mujer pobre, descansa... tal vez la que tenga plata. Porque uno de pobre...Que la barrida, que la trapiada, que la lavada, que la eche de comer, que despachar al marido "si tiene marido" si no los hijos, y busté se la pasa todo el día haciendo oficio y nunca termina... No nos metamos mentiras, ipa' nosotras las mujeres no hay descanso! Las ricas tal vez..."

"No pues que me daba ese dolor y. y... yo no le decía a la familia pues porque pues uno que necesita hacer el oficio y estar pendiente de la familia".

\section{Categoría 2. Asumiendo el rol de esposa, madre, abuela, suegra}

El mayor grupo de mujeres son viudas, seguido de separadas y solteras; carecen de una relación afectiva marital, así describen la experiencia:

"No sé o sería porque me agarró eso de... porque el papá de mis hijos me dejó, y usted sabe que uno, siempre sea como sea... yo tenía 18 años cuando me salí con él. ...él nunca me llegó a maltratar. Y que él me ponga personas y me las pase por encima...porque me decían que era la enfermedad de pena moral, que me había dado de pena moral porque yo pensaba mucho, yo lloraba mucho. ...Él vive ahí mismo, ahí enseguida de mi casa".

Las mujeres se desenvuelven cotidianamente con mayor participación en toma de decisiones de cualquier índole, con los diferentes miembros de la familia: hijos, nietos, nuera.

"Usted sabe que a esa edad una niña ya comienza ah... y si uno las deja, y si uno las deja entonces ya vienen es con su paquetico, con su tamalito $y$ entonces eso es lo que uno tiene que evitar (coloca las manos sobre el abdomen, simulando aumento de tamaño), y sonríe". 
"Si, emociones fuerte, sí porque son muy intensas, que si mi mamá si comió, que si el hijo que se me emborracha mucho, es muy peleonero, ya se emborracho, suena el teléfono ya me van a decir que está borracho, que para... y muy peleonero".

"Por ejemplo, que yo les diga: vea tal cosa, por ejemplo yo, yo lucho mucho con los niños. Yo estoy esperando que venga la madre, pa'entregárselos porque sinceramente, no. Ya no, hoy en día es muy difícil manejar la niñez, difícil, difícil. No cuando uno crió sus hijos, que yo crié mis hijos yo. No luchaba, yo no me pegaba la cabeza tanto con ellos".

"Suegra hágame una sopita de arroz, que le queda buena, entonces yo se la hice, me puse y se la hice. Cuando yo estaba haciéndola, trató como de darme el dolorcito. Cuando me agitaba mucho, o cuando yo alegaba así con la niña mía, o discutía con la nuera mía, o algo así..."

\section{Categoría 3. Apoyando a la familia y conviviendo con sus dificultades}

En algunas localidades de la región se vive en ambientes de incertidumbre ocasionándole a la mujer miedos, temores por la seguridad de su familia.

“...Y en parte lo que me dio... yo creo que la angustia porque mi hijo está preso, el robo, el atraco que me hicieron el domingo. El día anterior, que me que eso me atracaron ¡No!, no tengo, teléfono, no tengo cómo llamarles... quedé sola, nos desubicamos por esto, porque el teléfono como se lo...."

"Y de sobremesa el segundo día, dijeron que, que el hijo mío lo había levantado una persona de esos grupos, que yo no sé que, mentira no, todo era un equívoco, dijeron uno de esos grupos, de esos rastrojos, que no se que, se le alzaron a $X X X X$, se le alzaron a $X X X X$, imagínese como es eso, cómo no se me iba a agudizar eso..."

Las participantes manifiestan cómo parte del rol es tener la función protectora y cuando hay disfunción familiar se ve afectado su estado emocional;

“...algún alegato con los hijos me da, un desaliento en el cuerpo”.

“...Por ejemplo una mala noticia; yo tengo un hijo que llega tarde y yo le digo que maneje con mucho cuidado, y tengo un hijo que maneja su moto también y le digo que tenga mucho cuidado..."

Sin embargo, algunas participantes expresan que no todo es malo, estar acompañada y rodeada de la familia y de los vecinos se siente tener apoyo reciproco afectivo que además contribuyen y aportan con el cuidado de su salud.

"Mis hijos que están, los dos hijos que están aquí conmigo también ellos. Estaban pendientes de mí, y hasta ahora. Porque, pues ellos, ahora que estoy hospitalizada, ellos en la visita están aquí conmigo". 
"Yo estaba sola, como le digo, sola, yo salí así al balconcito y empecé a gritar; a llamar a los vecinos, a la vecina que quedaba en frente. ...Y entonces yo dije, así, como pude yo dije: ¡Por favor ayúdenme! ¡Por favor ayúdenme!”.

\section{Categoria 4. Interactuando con el entorno}

Esta categoría tiene relación con condiciones de vida, cómo influye su entorno social, sus fuentes de ingreso a nivel individual y familiar, la accesibilidad a los servicios de salud y la capacidad de respuesta que tienen de acuerdo con ciertas condiciones del ambiente físico como el calor, la luz, la humedad, la calidad del aire entre otros. Encontrándose las siguientes subcategorias:

\section{"VIVIENDO CON INCERTIDUMBRE POR LA INSEGURIDAD":}

Algunas de las participantes especialmente las de las áreas rurales expresan vivir enfrentadas a situaciones de violencia e inseguridad.

"Dijeron que... que el hijo mío lo había levantado una persona de esos grupos, que yo no sé que, mentira no, todo era un equívoco, antes fue que se le monto en una moto para irle a ayudar a llevar una llanta, y que no se qué, y dijeron uno de esos grupos, de esos rastrojos, que no sé qué, se le alzaron a Alexander, se le alzaron a Alexander, imagínese como es eso, (respiración profunda) cómo no se me iba a agudizar eso, bueno..."

"En nada, porque yo estaba dormida, desperté con ese ardor, no estaba pensando; Ah!! Fue un susto, ahora que me acuerdo fue un susto, yo estaba dormida, tranquila, cuando sentí unos pasos por el corredor, usted se imagina uno en una finca, y sentir unos, unos pasos por el corredor, y yo brinqué de una..."

\section{"REACCIONANDO A LOS CAMBIOS DE CLIMA":}

Cuando presenta síntomas la expresión más frecuente es la relacionada con la falta de aire y los cambios de temperatura.

"Yo no podía caminar ni una cuadra, que me daba ese cansancio y luego es como ese dolor, entonces yo me tenía que sentar por ahí en algún antejardín así y quedarme ahí hasta tomar aire. ... O sea aguanté, aguante, hasta cuando ya me dio fue el infarto de una, uno aguanta, ese cansancio de las piernas, como falta del aire, yo me salía a la ventana a tomar aire".

“Pues digo yo no,... me comenzó el dolor, y yo dije esto será por todo lo que me dijo esta señora, pero pues, en un brazo tenía que da' me para venirme, "yo no creía" yo ese dolor y ese dolor en el brazo, pero pues no fuerte yo llegaba y me envolvía un trapo que porque de pronto era de frio...".

\section{"POR LO QUE NO TENÍA PLATA PA' PAGAR":}

Es notable el déficit en el ingreso económico relacionado con las actividades que desarrollan las mujeres en el hogar sin ninguna remuneración y otras que laboran en el sector informal, la ausencia o carencia del dinero para suplir necesidades básicas 
del hogar lleva a las amas de casa a tener emociones con intensidad muy fuerte, generando conflicto interno y estrés.

"Si yo me desespero, porque por ejemplo, sabía yo que me venían a cobrar tal cosa y yo ahí dios mío que me vienen a cobrar, hay señor, sentía esa angustia como por tener pa' cubrir esa necesidad... ...Y el hijo mío que venía también con problemas económicos, entonces también me angustiaba, por no poderle servir, no puedo ayudarlo pa' quitarle ese problema de encima".

"Por lo que no tenía plata pa' pagar... pero si pienso mucho, esa casa que todavía no la he acabado de pagar, que se la quiten todo eso pienso, porque yo no he pagado esa casa".

"Que si no tengo trabajo, que sin un peso, porque es horrible uno sin un peso (llora), que necesitaba comprar a veces, a mi me dan la droga, pero a veces se me acababa"

"Me siento por ahí, y al ratico ya me paro a hacerles la comida a mis hijos "cuando tengo pues", porque cuando no tengo, no echo mano de nadies...

\section{“ACCEDIENDO A SERVICIOS DE SALUD CON DIFICULTAD":}

Acceder a los servicios de salud cuando ocurre la crisis en el momento de la urgencia las participantes expresan tres aspectos que son de gran importancia: El primero tiene que ver con la carencia de dinero pues manifiestan que los ingresos son insuficientes y en el momento que se requiera consultar de manera rápida o oportuna no siempre hay disponibilidad para cubrir los gastos del transporte, por tal motivo a veces hay retardo en consultar en caso de urgencia:

"Pues en el momento no tenía dinero para... para ir al médico, para... el transporte, pero lo conseguí, pero ya aquí en la clínica uno ya no da, de resto todo ha sido la clínica”.

El segundo aspecto es la dificultad que causa la condición geográfica, mujeres que viven en áreas rurales se desplazan desde sus viviendas hasta el centro de atención y luego de los municipios hasta la ciudad de Cali.

"Por ahí media hora... me llevaron en un taxi me parece que fue! No... en la finca, sube hasta cierta parte $y$ de ahí pa'lla uno no puede... va caminando...".

"Si de ahí me mandaron pa'ca, la enfermera me preguntó cómo me había dado, me tuvo un ratico sentada, y después si me hicieron acostar, y hay mismo la doctora vino y dijo que me tomaran un electro, hay mismo dijo que me trajeran para acá. En la ambulancia de Vijes, hasta aquí...”.

Si pertenecen al perímetro urbano también consultan primero al centro de salud de donde las remiten a un nivel mayor de referencia donde finalmente quedan hospitalizadas para el tratamiento.

"Entonces me trasladaron para $X X X$. No eso fue rapidito que me trajeron, el mismo día... Porque, es que allá no hay todos los recursos, allá no hay... 
...Claro! ¡Mami! Allá no hay los recursos..... No hay, mismo me trajeron en la ambulancia nada... nada... solo va remitida y ya! El médico le dijo esto a mi cuñada: "La vamos a remitir porque aquí no hay los recursos necesarios". Entonces, la, la, la doctora allá decía que no me podía recibir. Y volvía y me mandaba pa'allá, después volvieron y me mandaron. (Sonríe...) ija....jEstaban como haciendo paseo millonario! Después volvieron y me mandaron pa' allá pa' $X X X$, que no me recibían, que no me recibían... Que porque necesitaba un papel, ino sé qué papel! Hasta hoy no doy razón, pero yo no tenía dolor, nada, ya gracias a Dios. Entonces, allá me dejaron en los seguros y el día sábado ya me trajeron para acá, que desde eso hace que estoy aquí, día y noche me ponían droga, no sé qué droga sería... (Hace cara de desconocimiento). Porque, pa' qué ¡bien atendida si estoy! Gracias a Dios”.

Aaahhh, jsí!, al hospital. Si yo he ido varias veces pero por el dolor del pecho, yo he ido por urgencias y me toman un electro me dicen ya... ya está bueno, pero esta vez me remitieron. Me llevaron al hospital, me vi fue ahí en Carlos Holmes. Y de allí me tomaron un, un, un ¿Cómo es que se llama? No del corazón, no del corazón, no el de allá del Carlos Holmes. Un electro, me tomaron un electro. Entonces ya el médico pidió pues, acá pà que me remitieran pa cá pa`l departamental”.

El tercer aspecto es que a pesar de expresar las incomodidades mencionadas con anerioridad, finalmente las participantes reconocen que al llegar a las instituciones sienten que han sido atendidas de inmediato y haber recibido la atención que requieren, realizándoles valoración, las ayudas diagnósticas y administrando tratamiento de emergencia.

"Al hospital Carlos Carmona...Entonces el doctor me puso una pastica debajo de la lengua (se toca con el dedo índice la parte sublingual), y me colocaron una inyección en la vena. Cuando volvió y me dió, yo dejé de almorzar y me paré y ay Dios mío, me dijo: tómese otra pasta...Pásela a ella rápido a urgencias que tiene un dolor no se qué, y la enfermera me llevó y me...allá llegó un doctor y me vio de una".

"Entonces me dijo la señorita, que tiene, le dije no vengo muy mala, vengo muy mala (mueve sus brazos entrecruzándolos continuamente) y ella misma le dijo a mi hijo: no, no presente papeles venga, venga, venga con ella primero y me entró donde una doctora de turno, me acostaron, ahí me quedé quietica (extiende sus brazos sobre sus piernas), la doctora me tomó el electro, oí que dijo que tenía un Infarto".

\section{Categoría 5. Cumpliendo con el tratamiento}

ADHERENCIA A TRATAMIENTO PREVIO, BAJO CONTROL MÉDICO: "Yo tomo porque a mí me las manda el médico"

"¡He estado 6 meses!... A mí me habían mandao.... nitrato de isordil debajo de la lengua media pastillita. Hasta hace poco eso era lo que le calmaba". Sí, yo me lo puse debajo de la lengua. ¡No me calmó!”.

"Es como cuando yo fui al médico, me mandó unas pastilla y yo podía decir que de pronto esas pastillas me, me dio eso. Porque, esas pastillas me 
asentaron mal y yo las... me alcancé a tomar ocho. Yo dejé esas pastas por ahí, de allí p’a acá ese dolor. Y no sé, ahí en casa están esas pastillas, no sé allí en casa están las pastillas".

TRATAMIENTO SIN FORMULACIÓN: “...Me tomo por ahí cualquier pasta”

"Hay veces que me tomo por ahí cualquier pasta. No... yo, tomo ese dolex. Entonces yo no fui... y ahí me aguanté mi dolor, me tomaba pastas, me tomaba...lbuprofeno... eso no me valía”.

Su compromiso y cumplimiento con la prescripción médica ayudan a mejorar sus síntomas, evitar complicaciones y hospitalizaciones.

\section{DISCUSIÓN}

La responsabilidad laboral y necesidad de remuneración hace que continúen trabajando con el disconfort interpretándose dicha situación como la "minimización" del problema. Estas manifestaciones son consideradas como una estrategia cognitiva según muestran los estudios acerca del afrontamiento, su estado máximo produce perturbación, consulta a un experto, "búsqueda de ayuda instrumental", considerada como estrategia comportamental ${ }^{(27)}$. Incluso algunas de las mujeres dejan de trabajar o modifican sus actividades siempre relacionadas con el "rol de amas de casa" confinadas al servicio doméstico lo que ellas denominan "tener que hacer el oficio de la casa". Históricamente en la regulación del trabajo a las mujeres se le asignaron los trabajos menos calificados, como el trabajo doméstico, especializándose cada vez más en la atención a las necesidades de la familia, en algunos casos sin retribución económica y en otras situaciones con salarios inferiores ${ }^{(28)}$.

La mayoría carece de una relación afectiva marital, ante la separación asumen el rol de "Mujer cabeza de hogar", suplen las carencias económicas y afectivas estrechando vínculos y reconstituyendo las familias extensas con mayor participación en toma de decisiones de cualquier índole, con los diferentes miembros de la familia.

En caso de separación se genera un clima tensional, como se encontró en un estudio donde se analizaron las características psicológicas con las diferencias de género, en sujetos internados en una unidad coronaria ${ }^{(29)}$. Asumir el rol de "Mujer cabeza de hogar", suplir las carencias económicas y afectivas conlleva a estrechar vínculos reconstituyendo las familias extensas con mayor participación en toma de decisiones de cualquier índole, con los diferentes miembros de la familia.

Un estudio retrospectivo mostró aumento significativo de riesgo relativo, e incidencia de angina de pecho cuando las personas estaban expuestas a ansiedad, hiperlipidemia y problemas psicosociales familiares, así lo muestra el que la mayoría de las participantes se desenvuelven en entornos donde los conflictos sociales y familiares por resolver producen en ellas respuestas emocionales percibidas como potencialmente estresantes ${ }^{(30)}$; situación similar se encontró en estudio que se realizó años anteriores en una de las instituciones donde se desarrolló la presente investigación, cuyo objetivo fue evaluar factores de estrés y apoyo social en personas mayores con antecedentes de infarto agudo de miocardio ${ }^{(31)}$. Pero, también expresan que pertenecer a un contexto familiar y comunitario favorece positivamente, viéndolo como un recurso de apoyo social ${ }^{(27) .}$ 
Respecto a la interacción con el entorno debido a la presencia de grupos al margen de la ley, la violencia e inseguridad ha generado en las comunidades que vivan permanentemente con miedo e incertidumbre.

Carencia del dinero para suplir necesidades básicas del hogar lleva a las amas de casa a tener emociones con intensidad muy fuerte, generando conflicto interno y estrés, obligándolas a consultar a las instituciones de nivel I de complejidad hasta llegar a instituciones nivel III y IV además de los requisitos administrativos para hospitalización y autorizaciones de estudios y tratamiento ocasiona incomodidades. En una de las entidades se realizó un estudio durante los años 2003 y 2004 el cual reporta $38.5 \%$ de procedimientos realizados por la especialidad de cardiología intervencionista entre arteriografías y angioplastia coronaria, fue realizado a mujeres desde los 30 años de edad ${ }^{(32)}$, con alto riesgo de enfermar o de morir, que pueden conducir a IAM ocasionando complicaciones crónicas que afectan de modo definitivo la calidad de vida de la persona así como el daño psicológico y social causado por enfermedad coronaria, como es el caso de las mujeres participantes que denota como no se apartan de dicha realidad.

Se denota una respuesta al entorno ambiental, también manifiestan tener respuesta físicas de disconfort respecto a los cambios en el clima que tiene similitud con el estudio realizado en Girardot-Colombia ${ }^{(26)}$, cuyo contexto geográfico, clima y temperatura son similares al municipio de Cali, ambas regiones ribereñas.

El estudio de autocuidado y adherencia en pacientes con falla cardiaca ${ }^{(33)}$ hace referencia a que para una mejor adherencia al tratamiento farmacológico y no farmacológico, se requiere de intervención educativa que ayuda a los pacientes a superar el déficit de conocimiento, conocer los medicamentos, la importancia de la enfermedad, la dieta, mantener el peso adecuado, reconocer signos y síntomas, realizar ejercicio, aceptación de la enfermedad y tener confianza en el profesional de salud.

Integrar en el cuidado de enfermería el enfoque de género por condiciones de equidad y de igualdad en pro del bienestar y del cuidado de la salud de la mujer considerando el rol que históricamente ha desempeñado. Las redes de apoyo a nivel comunitario deben tener una formalidad e integrarse con el trabajo de la enfermería en el cuidado de los colectivos considerándolo como fuente proveedora para la seguridad, el cuidado y la convivencia en el entorno.

Desde los servicios de salud se debe brindar mayor apoyo a las mujeres con enfermedad isquémica a través de la consejería que sea ofertada por profesionales de enfermería, permitiendo una mayor y mejor relación terapéutica, de escucha, que la mujer se sienta visibilizada en los servicios de salud comprendida como un todo en la dimensión humana.

\section{CONCLUSIONES}

Lo que Lenz y col. describe como factores determinantes en las mujeres propias del contexto, las del Valle del Cauca, tienen relación con antecedentes de tipo fisiológico, pero más influye el rol que la mujeres asume y desempeña en sus propias dinámicas familiares que son el eje y soporte de familia nuclear y extensa inclusive, no solo en lo afectivo sino también en lo económico, sumado a esto que se evidencia las condiciones de desventajas en cuanto al panorama laboral se refiere. 
El rol que la mujer ha desempeñado históricamente en nuestra sociedad, en condiciones de desigualdad, asimetría y sumisión respecto al hombre sigue evidente y es reflejado en el modo de vida en condiciones de vulnerabilidad a la mujer, en cuanto al concepto de bienestar se refiere. Se sugiere profundizar desde otras metodologías, investigación e intervención para aportar en la negociación y modificación de aquellos determinantes sociales y culturales. Teniendo en cuenta el rigor de aplicabilidad y transferibilidad, considerando los resultados del estudio que es Multicéntrico.

\section{REFERENCIAS BIBLIOGRÁFICAS}

1. Mendis, S.; Puska, P.; Norrving, B.; World Health Organization, Global atlas on cardiovascular disease prenvention and control. World Health Organization, 2011). [on line]. [citado 2013 may 02]. Disponible en http://apps.who.int/iris/handle/10665/44701

2. Alwan, A. World Health Organization. Global status report on noncommunicable diseases 2010, 2011, [on line]. [citado 2013 may 03]. p. 176Disponible en: http://whqlibdoc.who.int/publications/2011/9789240.

3. 3. World Health Organization Mortalidad debida a Enfermedades Cardiovasculares en las Américas. [on line]. [citado 2013 may. 03]. Disponible en http://www.paho.org/hipertension/?page_id=298

4. Organización Mundial de la Salud. Enfermedades cardiovasculares, Enfermedades no transmisibles y salud mental... [on line]. [citado 2013 abr. 02]. Disponible en: http://www.who.int/mediacentre/factsheets/fs317/es/index.html

5. Departamento Administrativo Nacional de Estadística. Defunciones según causas agrupadas de defunción 667-OPS-CIE-10. [on line]. [citado 2011 oct. 30]. Disponible en https://www.dane.gov.co/...y...defunciones/nacimientos-ydefunciones

6. Enfermedad cardiovascular: principal causa de muerte en Colombia Boletín No. 1, Diciembre 9 de 2013. [on line]. [citado 2014 may 30]. Disponible en http://www.ins.gov.co/lineas-deaccion/ons/boletin\%201/boletin web ONS/boletin 01 ONS.pdf)

7. Departamento Administrativo Nacional de Estadística. Comité Departamental Estadísticas Vitales en el Valle del Cauca. Censo y proyecciones basadas en el censo año 1993. Mortalidad: Primeras causas total general 2005, Valle del Cauca. [on line]. [citado 2011 nov. 12].

En http:// www.valledelcauca.gov.co/salud/descargar.php?id=1399

8. Gobernación del Valle del Cauca. Secretaria Departamental de Salud. Plan Departamental de Salud 2008-2011. [on line]. [citado 2011 oct. 31].Disponible en www.valledelcauca.gov.co/salud/descargar.php?id=11757

9. CEDETES - Universidad del Valle/ Secretaria de salud Pública Municipal de Cali, (2007). Situación de Salud en Santiago de Cali - Una mirada desde la salud pública. 2007 abr. [citado 2011 feb. 02]; Disponible en http://new.paho.org/hq/dmdocuments/2010/FESP_Modelo_Salud_Publica_ColombiaCali-2007.pdf

10. Asociación Latinoamericana de Medicina Social. Taller Latinoamericano de Determinantes Sociales de la Salud. 2008 dic. 07 [citado 2011 mar 02]; Disponible en htpp://www.alames.org/.../materiales-taller-determinantes-sociales/ 
11. Jaramillo N., Torres Y. Aspectos epidemiológicos de la enfermedad cardiovascular: factores de riesgo a la luz de Framingham. CES MEDINA. [revista en la Internet]. 2004 jul-dic [citado 2011 abr. 13]; 18(2): 45-68. Disponible en http://wwwrevistas.ces.edu.co/index.php/medicina/article/download/478/274.

12. Gómez Rubén. La noción de "Salud Pública": consecuencias de la polisemia. Facultad Nacional de Salud Pública [revista en la Internet]. 2002 [citado 2011 feb 04]; 20(1):101-116. Disponible en: http://www.redalyc.org/resumen.oa? id =12020109

13. Gómez Elsa. Equidad, género y salud: retos para la acción. Panamericana de Salud Pública [revista en la Internet]. 2002 [citado 2012 ene 9]; 11(5-6): 454-461 Disponible en http//:www.scielosp.org/scielo.php?pid=S1020-49892002000500024

14. Barbary O, Urrea F. Gente Negra en Colombia. Dinámicas sociopolíticas en Cali y el Pacífico. Ed. Centro de Investigaciones y Documentación Socioeconómica de la Facultad de Ciencias Sociales de la Universidad del Valle Cali; 2004.

15. Guevara Rubén D. Violencia y desplazamiento: caracterización de las mujeres desplazadas jefes de hogar del municipio de Florida, Valle del Cauca. Reflexión Política [revista en la Internet]. 2008 jun [citado 2011 abr 9]; 10(20): 154-173. Disponible http://revistas.unab.edu.co/index.php?journal=reflexion\&page=article\&op=viewArticle\& path $\% 5 \mathrm{~B} \% 5 \mathrm{D}=520$

16. Ortiz C., Uribe J., García G. Informalidad y Subempleo: Un Modelo Probit Bivariado aplicado al Valle del Cauca. Sociedad y Economía Univalle [revista en la Internet]: 2007 [citado 2010 abr 28]; 13(9-10). Disponible en http://dintev.univalle.edu.co/revistasunivalle/index.php/SyE/article/view/145

17. Urrea F., Puerto Fernando. Expresiones de religiosidades populares y prácticas de salud en un área urbana de Cali (Colombia). Signo y Pensamiento [revista en la Internet]. 2000 [citado 2011 oct. 31]; XIX (37): 39-48. Disponible en: http//www.javeriana.edu.co/signoyp/coleccion.htm

18. Lenz E., Pugh I., y col. La teoría de rango medio de los síntomas desagradables: Una actualización. Adv Nurs Sci [revista en la Internet] 1997 Mar [citado 2011 ago 31]; 19(3):14-27. Disponible en http//www.ncbi.nlm.nih.gov/pubmed/9055027

19. Krippendorff K. Metodología de análisis de contenido, teoría y práctica. Ed. Paidós Iberoamérica S.A. Barcelona, 1997.

20. Navarro P, Díaz, C. Delgado J. Análisis de Contenido. Métodos y técnicas cualitativas de investigación en ciencias sociales. Editorial SINTESIS. Tercera reimpresión. 1999.

21. Castillo E., Vásquez M.L. El rigor metodológico en la investigación cualitativa Colomb. Med.

[revista en la Internet]. 2003 [citado 2011 ene 08]; 34(3):164-167. Disponible en http://www.redalyc.org/articulo.oa?id=28334309

22. República de Colombia. Resolución No. 008430 de 1993 del Ministerio de Salud. [citado 2011 feb 05]. Disponible http://www.urosario.edu.co/urosario_files/a2/a24fb07a-f561-4fcc-b611affff4374bb7.pdf

23. MORSE Janice. Asuntos críticos en los métodos de investigación cualitativa. Medellín: Editorial Universidad de Antioquia. Colección Contus; 2003.

24. Cabero J., Loscertales F. Elaboración de un sistema categorial de análisis de contenido para analizar la imagen del profesor y la enseñanza en la prensa. Rev. Bordón [revista en la Internet]. 1996 [citado 2010 abr. 20]; 48(4):375-392

Disponible en http://tecnologiaedu.us.es/cursos/32/html/bibliovir/8.htm

25. Coffey A., Atkinson P. Encontrar el sentido a los datos cualitativos. Estrategias complementarias de investigación. Medellín Editorial Universidad de Antioquia. Colección Contus. 2003. 
26. Cortés Luis Alberto. Tipificación del síntoma dolor torácico tipo isquémico en la mujer, a la luz de la teoría de los síntomas desagradables. Avances en enfermería [revista en la Internet] 2007 [citado 2011 abr. 03]; 25(2):76-89. Disponible en http://www.enfermeria.unal.edu.co/revista/articulos/xxv2_8.pdf

27. Rodríguez J., Pastor M.A., López R. Afrontamiento, apoyo social, calidad de vida y enfermedad. Red de Revistas Científicas de América Latina y el Caribe, España y Portugal.. Psicothema [revista en la Internet] 1993 [citado 2011 feb 24]; 5, (supl): 349-372. En Disponible en: htpp://www.redalyc.org/articulo.oa?id=72709924

28. Posso Jeanny. La inserción laboral de las mujeres inmigrantes negras en el servicio doméstico de la ciudad de Cali. Editorial: Universidad del Valle. 2008. [citado 2011 feb 24];

Disponible en htpp://programaeditorial.univalle.edu.co/index.../virtuemart/?page...

29.Urquijo S., Giles M., R ichard's, M., Pianzolas E. Variables psicológicas asociadas a las patologías isquémico-cardiovasculares. Un estudio de género. Rev Argentina, Cardiología [revista en la Internet] 2003 [citado 2011 mar 04]; 32: 190-198.

Disponible en http://www.fac.org.ar/1/revista/03v32n2/artorig/arorig02/urquijo.PDF 30. Medalie JH., Goldbourt U. La angina de pecho entre los 10.000 hombres. II. Factores psicosociales de riesgo y otros, como lo demuestra un análisis multivariado de un estudio de incidencia de cinco años. Revista » The American journal of medicine. [revista en la Internet] 1976 May 31 [citado 2011 mar 04]; 60(6):910-21. Disponible en http://www.ncbi.nlm.nih.gov/pubmed/798490

31. Reyes C., Hincapié M., Herrera J., Moyano P. Factores de estrés y apoyo psicosocial en pacientes con infarto agudo de miocardio. Cali, 2001-2002. Rev. Colombia Médica [revista en la Internet]. 2004 [citado 2011 abr 17]; 35(4): 199-204 Disponible

en

http://colombiamedica.univalle.edu.co/index.php/comedica/article/viewArticle/319

32. Rodríguez Hernán. Gasto por procedimiento en la especialidad de cardiología intervencionista en pacientes con enfermedad coronaria del Seguro Social, Seccional Valle del Cauca. Noviembre 2003-diciembre 2004. Rev. Colombia Médica [revista en la Internet]. oct.-dic. 2006 [citado 2011 may 02]; 37(4): 266-274. Disponible en http://colombiamedica.univalle.edu.co/index.php/comedica/article/viewArticle/456/1042 33. Achury Diana M. Auto cuidado y adherencia en pacientes con falla cardiaca. AQUICHAN, [revista en la Internet]. 2007 [citado 2012 sep. 12]; 7(002): 139-160 2007.

Disponible en http://www.scielo.unal.edu.co/scielo.php?script=sci_arttext\&pid=S1657$59972007000200004 \&$ Ing $=$ es\&nrm=

Recibido: 11 de Septiembre 2014; Aceptado: 10 de Octubre 2014

ISSN 1695-6141

๑ COPYRIGHT Servicio de Publicaciones - Universidad de Murcia 